ARTICLE

\title{
Femtosecond X-ray emission study of the spin cross-over dynamics in haem proteins
}

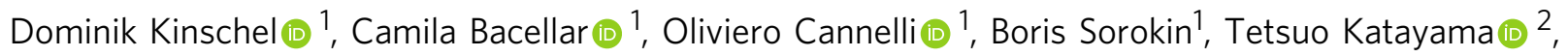
Giulia F. Mancini (1) ${ }^{1}$, Jérémy R. Rouxel ${ }^{1}$, Yuki Obara ${ }^{3}$, Junichi Nishitani ${ }^{4}$, Hironori 1 Ito $^{3}$, Terumasa Ito $^{3}$, Naoya Kurahashi ${ }^{5}$, Chika Higashimura ${ }^{4}$, Shotaro Kudo ${ }^{4}$, Theo Keane (i) ${ }^{6}$, Frederico A. Lima7, Wojciech Gawelda (1) 7,8, Peter Zalden77, Sebastian Schulz7, James M. Budarz (1) 1, Dmitry Khakhulin7, Andreas Galler ${ }^{7}$, Christian Bressler ${ }^{7}$, Christopher J. Milne (i) ${ }^{9}$, Thomas Penfold ${ }^{6}$, Makina Yabashi (i) ${ }^{2}$, Toshinori Suzuki (D) ${ }^{4}$, Kazuhiko Misawa ${ }^{3}$ \& Majed Chergui (iD ${ }^{1 凶}$

In haemoglobin the change from the low-spin (LS) hexacoordinated haem to the high spin (HS, $\mathrm{S}=2$ ) pentacoordinated domed deoxy-myoglobin (deoxyMb) form upon ligand detachment from the haem and the reverse process upon ligand binding are what ultimately drives the respiratory function. Here we probe them in the case of Myoglobin-NO (MbNO) using element- and spin-sensitive femtosecond $\mathrm{Fe}_{\alpha}$ and $\mathrm{K}_{\beta} \mathrm{X}$-ray emission spectroscopy at an X-ray free-electron laser (FEL). We find that the change from the $L S(S=1 / 2) \mathrm{MbNO}$ to the HS haem occurs in $~ 800 \mathrm{fs}$, and that it proceeds via an intermediate $(S=1)$ spin state. We also show that upon NO recombination, the return to the planar MbNO ground state is an electronic relaxation from $\mathrm{HS}$ to LS taking place in $\sim 30 \mathrm{ps}$. Thus, the entire ligand dissociation-recombination cycle in $\mathrm{MbNO}$ is a spin cross-over followed by a reverse spin cross-over process.

\footnotetext{
${ }^{1}$ Laboratoire de Spectroscopie Ultrarapide (LSU) and Lausanne Centre for Ultrafast Science (LACUS), Ecole Polytechnique Fédérale de Lausanne (EPFL), 1015 Lausanne, Switzerland. ${ }^{2}$ Japan Synchrotron Radiation Research Institute (JASRI), 1-1-1, Kouto, Sayo-cho Sayo-gun, Hyogo 679-5198, Japan. ${ }^{3}$ Tokyo University of Agriculture and Technology (TUAT), 2-24-16 Naka-cho, Koganei, Tokyo 184-8588, Japan. ${ }^{4}$ Department of Chemistry, Graduate School of Science, Kyoto University, Kitashirakawa-Oiwakecho, Sakyo-Ku, Kyoto 606-8502, Japan. ${ }^{5}$ Department of Materials and Life Sciences, Faculty of Science and Technology, Sofia University, 102-8554 Tokyo, Chiyoda, Kioicho 7-1, Japan. ${ }^{6}$ Chemistry-School of Natural and Environmental Science, Newcastle University, Newcastle upon Tyne NE1 7RU, UK. ${ }^{7}$ European XFEL, Holzkoppel 4, 22869 Schenefeld, Germany. ${ }^{8}$ Faculty of Physics, Adam Mickiewicz University, 61-614 Poznań, Poland. ${ }^{9}$ SwissFEL, Paul-Scherrer-Institut (PSI), 5232 Villigen, Switzerland. ${ }^{凶}$ email: majed.chergui@epfl.ch
} 
$\int$ mall ligand molecules $\left(\mathrm{O}_{2}, \mathrm{CO}, \mathrm{NO}, \mathrm{CN}\right.$ and $\left.\mathrm{H}_{2} \mathrm{O}\right)$ are the receptors that bind to and activate haem proteins, such as myoglobin $(\mathrm{Mb})$ and haemoglobin $(\mathrm{Hb})$. Their active site (Fig. 1) is a porphyrin with an Iron ion (that can be either ferric$\mathrm{Fe}^{3+}$ or ferrous- $\mathrm{Fe}^{2+}$ ) at its centre to which the ligands bind on the distal side. The haem itself is linked to the $\mathrm{F}$ helix of the protein via a histidine (His) amino-acid bound to the $\mathrm{Fe}$ ion on the proximal side of the porphyrin. The ferrous forms of $\mathrm{Mb}$ and $\mathrm{Hb}$ play an essential role in respiration via the transport and storage of oxygen in mammals. In the ligated form, the hexacoordinated $\mathrm{Fe}^{2+}$ ion of the haem is in a low-spin (LS) planar singlet $(\mathrm{MbCO})$ or doublet state $(\mathrm{MbNO})$. Upon release of the ligand from the $\mathrm{Fe}^{2+}$ ion, the haem switches to a high-spin (HS) quintet state with the pentacoordinated $\mathrm{Fe}$ in an out-of-plane, socalled domed, deoxyMb configuration (Supplementary Fig. 1). The shuttling between the LS planar to HS-domed spin cross-over (SCO) process defines the "transition state", which governs the allosteric transitions in haem proteins (including $\mathrm{O}_{2}$ carriers and $\mathrm{NO}$-sensor and CO-sensor) $)^{1,2}$. In particular it is the first event of the respiratory function in which, after release of the distal ligand, the $\mathrm{Fe}$ atoms moves out of the porphyrin plane, pushing the proximal His linked to the F helix and displacing the latter (Fig. 1). This is considered the step by which the monomer units of $\mathrm{Hb}$ transfer information, ultimately leading to the transition from the so-called "relaxed" to "tense" state at later times $^{3}$.

It has long been known that ligand detachment in ferrous Mb's can be induced by visible-ultraviolet (UV) excitation of $\pi-\pi^{*}$ transitions of the haem ${ }^{4}$. This has been exploited to mimic the release of the distal ligand from the Fe centre, and monitor its recombination to the haem. With the advent of ultrafast spectroscopy, haem proteins were among the first systems ever to be studied, specifically with the aim of monitoring these processes in "real-time" using transient absorption (TA) spectroscopy from the visible-UV (sensitive to the porphyrin $\pi-\pi^{*}$ transitions) $)^{5-9}$ to the Infrared (IR) (sensitive to the ligand stretch and the porphyrin vibrational modes $)^{10,11}$, as well as by time-resolved resonance Raman spectroscopy (sensitive to the $\mathrm{Fe}-\mathrm{His}$ bond) ${ }^{12,13}$. These studies concluded that photodissociation of the diatomic ligands and doming are prompt and simultaneous events, typically in $<50-100$ femtoseconds (fs) ${ }^{14}$. The actual formation of the ground state HS deoxyMb form has been described by two main and non-mutually excluding models: (a) a vibrationally hot ground-state is formed promptly after excitation, in which cooling ensues $8,15,16$ and (b) the process is a cascade through intermediate electronic excited states ${ }^{6,17}$.
Recently, the occurrence of prompt doming was questioned in a fs hard X-ray absorption spectroscopy (XAS) study of photoexcited $\mathrm{MbCO}^{18}$. Time-resolved XAS is an ideal element-selective probe of the electronic and geometric structure changes of metalcontaining molecular complexes ${ }^{19}$. Using a monochromatic probe at $7.123 \mathrm{keV}$ (tuned to the Fe K-edge), Levantino et al. 18 observed a first $<50-70 \mathrm{fs}$ event, which they attributed to $\mathrm{CO}$ photolysis from the LS $(S=0) \mathrm{MbCO}$ and partial SCO to a higher spin state, followed by a further $300-400 \mathrm{fs}$ event, attributed to the passage to the HS $(S=2)$ state of deoxyMb. This picture was supported by quantum wave packet dynamics simulations ${ }^{20}$, which accounted for coherent nuclear and electronic motions. However, Levantino et al.'s ${ }^{18}$ use of a monochromatic probe hindered a spectroscopic identification of the intermediate and final states, let alone of their spin. In recent years, non-resonant $\mathrm{X}$-ray emission spectroscopy (XES) has emerged as a valuable tool to identify spin states of transition metal complexes (TMC) ${ }^{21}$. The $\mathrm{K}_{\beta 1,3}$ and $K_{\beta}^{\prime}$ lines $(3 p \rightarrow 1 s$ emission, Fig. $2 \mathrm{a})$ have been used as markers of the spin state (number of unpaired $3 d$ electrons) of TMC's via the $3 p-3 d$ exchange interactions and fs transient $\mathrm{K}_{\beta}$ XES was successfully implemented to monitor the SCO dynamics in $\left[\mathrm{Fe}(\mathrm{bpy})_{3}\right]^{2+22}$, as well as to identify the HS deoxy haem product of photoexcited ferrous cytochrome $\mathrm{c}$ after dissociation of its distal methionine ligand ${ }^{23}$. In the case of $2 p$-orbitals, the $p-$ $d$ exchange is weaker but $\mathrm{K}_{\alpha}$ XES $(2 p \rightarrow 1 s$ transitions, Fig. $2 \mathrm{a})$ can also be used as a marker of spin state via the linear dependence of the full-width at half-maximum (FWHM) of the $\mathrm{K}_{\alpha 1}$ line as a function of the number of unpaired metal $3 d$ electrons ${ }^{24}$.

In this contribution, we focus on the case of nitrosylmyoglobin (MbNO). The role of nitric oxide (NO) in physiological processes in humans was discovered in the $1990 \mathrm{~s}^{25}$. In its interaction with haem systems, the NO molecule plays an important role in several physiological effects and biological functions, such as neurotransmission ${ }^{26}$, regulation of vasodilatation ${ }^{27}$, platelet aggregation $^{28}$, and immune response ${ }^{29}$. Furthermore, studies of NO release from and binding to the haem provide mechanistic information of relevance for other ligands active in respiration, blood pressure regulation, nitrogen fixation, and $\mathrm{NO}$ oxidation. In this respect, several time-resolved studies using visible-UV and IR probes have been carried out on $\mathrm{MbNO}$ over the past three decades. Just as for MbCO, NO photodissociation from the haem has been reported to be prompt ( $<100 \mathrm{fs}$ ) with a quantum yield of $\sim 55 \%{ }^{7}$, and recovery of the system occurs on typical timescales of $1-3,5-20,110-290 \mathrm{ps}$, and a weak ( 10-20\%) ns component ${ }^{7,11,13,30-37}$. The shortest recovery time was attributed to cooling within what was considered, the promptly formed hot

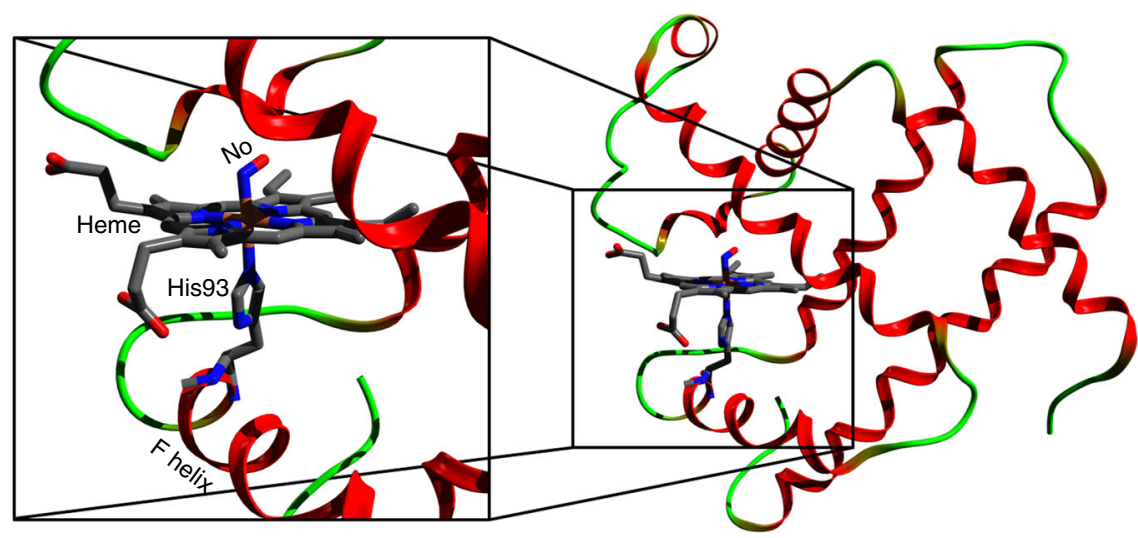

Fig. 1 Crystal structure of the nitrosyl-myoglobin (MbNO) (PDB entry: 10.2210/pdb1hjt/pdb). The haem is represented as sticks (Fe [orange], C [grey], $\mathrm{N}$ [blue], $\mathrm{O}$ [red]). The zoom (left) shows the distal NO ligand and proximal histidine 93 (His93), which links the Fe atom of the haem porphyrin to the F helix. 
a

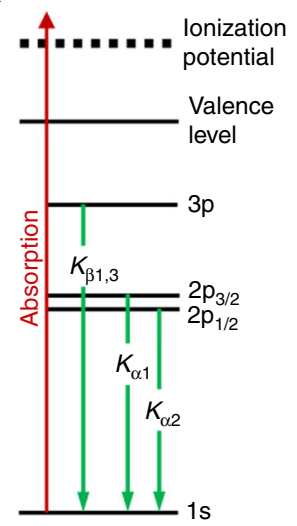

b

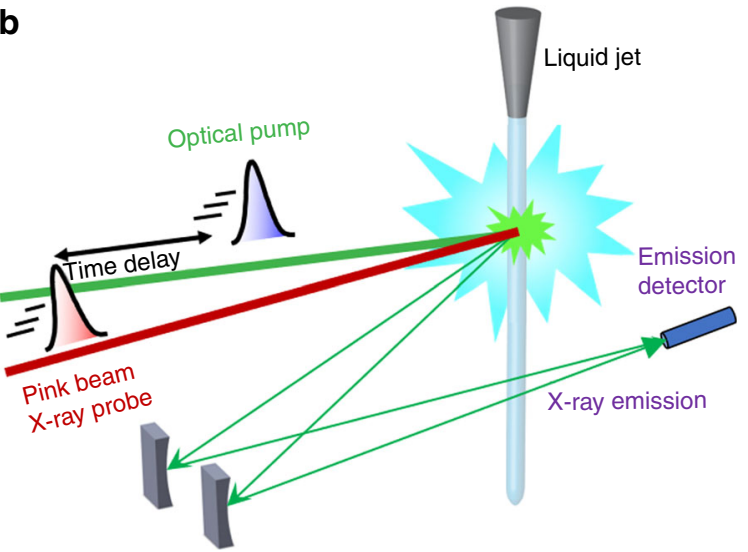

Fig. 2 Experimental scheme. a Energy level diagram showing the origin of the $K_{\alpha}$ and $K_{\beta}$ fluorescence after creation of a hole in the $1 s(K)$ shell. The $K_{\alpha 1}$ and $K_{\alpha 2}$ lines originate from the splitting of the $2 p$ orbital $\left(2 p_{1 / 2}\right.$ and $\left.2 p_{3 / 2}\right)$, whereas for $K_{\beta}$ these lines are degenerate, resulting in the line labelled $K_{\beta 1,3}$. b Experimental setup for the time-resolved X-ray emission spectroscopy measurements at the XFEL. The green line represents the optical (533 nm) pump pulse used to excite the sample and the red line represents the $\mathrm{X}$-ray (centred at $8.168 \mathrm{keV}$ ) probe pulse at varying time delays (black arrow). The pulses are overlapped and intercept the sample at the green interaction region. A von Hamos geometry was used for these measurements.

ground state of deoxyMb ${ }^{8}$, while the next two decay times were attributed to geminate recombination to the latter of, respectively, NO ligands from the distal pocket close to the haem and NO ligands from the more distant Xe4 pocket. Finally, the nanosecond (ns) component is due to non-geminate recombination of a small fraction of NO ligands that have escaped the protein. Contrary to other ligands ( $\mathrm{CO}$ in particular), which can bind to a planar haem, the unpaired NO electron allows its binding to the domed HS deoxyMb ${ }^{36}$, and this was recently supported by a time-resolved $\mathrm{Fe} \mathrm{K}$-edge X-ray near edge structure (XANES) study with $70 \mathrm{ps}$ resolution ${ }^{37}$. The binding of NO to a domed deoxyMb (hereafter designated as deoxyMb-NO) had previously been predicted by theoretical calculations on model haems ${ }^{38}$, which argued that the return to the planar form is a reverse SCO from $\mathrm{HS}$ deoxyMb-NO to LS planar MbNO. Recent ultrafast resonance Raman studies ${ }^{13}$ monitoring the Fe-His bond (Fig. 1) determined that the domed-to-planar transition in MbNO takes place in $\sim 30 \mathrm{ps}$ and argued that the process is determined by constraints exerted by the protein structure on the haem cofactor (Fig. 1). Since the entire ligand dissociation-recombination cycle occurs within $\sim 250 \mathrm{ps}$, and is much shorter than the commonly studied $\mathrm{MbCO}$, this implies that it can be monitored within the same ultrafast spectroscopic experiment.

In order to identify the mechanisms leading to the formation of $\mathrm{HS}$ deoxyMb in myoglobins and its time scale, and the specific case of the reverse deoxyMb to $\mathrm{MbNO}$ transition, here we use fs Fe $K_{\alpha}$ and $K_{\beta}$ non-resonant XES with a von Hamos spectrometer (Fig. $2 \mathrm{~b}$ ) at the SPring-8 angstrom compact free electron laser (SACLA) $^{39,40}$. We record laser-off and laser-on XES spectra (whose difference yields the transient XES) as a function of time delay with respect to the pump pulse. We find that upon $533 \mathrm{~nm}$ photoexcitation of $\mathrm{MbNO}$ into the Q-band (Supplementary Fig. 2), the SCO from the ground state LS $(S=1 / 2) \mathrm{MbNO}^{41}$ to the HS $(S=2)$ deoxyMb occurs in $\sim 800 \mathrm{fs}$ and involves the passage via an intermediate $S=1$ spin state, while the return from the HS deoxyMb-NO to the LS MbNO is a reverse SCO that takes place in $\sim 30 \mathrm{ps}$. We thus establish the details of domed HS deoxyMb formation, which we argue is of general validity to all $\mathrm{Mb}$ 's, and we identify the return to the initial $\mathrm{MbNO}$ ground state also as a HS-LS relaxation. The observation of the $S=1$ state is supported by density functional theory (DFT) calculations of the $\mathrm{K}_{\alpha}$ XES. Details of the experimental set-up and procedures and of the theoretical calculations are given in the SI.

\section{Results and discussion}

X-ray emission transients. In previous ultrafast ${ }^{22,23}$ or quasistatic $^{42} \mathrm{~K}_{\beta}$ XES studies, the assignment of spin states relied on the comparison with reference spectra of model compounds. Supplementary Fig. 3 shows the steady-state $K_{\beta}$ emission spectra of hexacoordinated $\mathrm{MbNO}$ and pentacoordinated deoxyMb and compares them with those of reference Fe-based molecular complexes in Supplementary Fig. $3 b^{22}$, and reference Fe(II) LS and HS porphyrin compounds ${ }^{42}$. While the trends are similar as a function of increasing the spin in all three panels, with the intensity increase of the $\mathrm{K}_{\beta}$, line around $7045 \mathrm{eV}$ and a blue shift and intensity decrease of the $\mathrm{K}_{\beta 1,3}$ line near $7058 \mathrm{eV}$, some differences also show up in that the blue shift of the $K_{\beta 1,3}$ line in Supplementary Fig. $3 \mathrm{a}$ and its energy splitting with respect to the $\mathrm{K}_{\beta}$, line are smaller than in Supplementary Fig. $3 \mathrm{~b}$ and c. These differences may point to a covalency change ${ }^{43-46}$ in addition to the spin change, as will be discussed later. Supplementary Fig. 4a shows the steady-state $\mathrm{K}_{\alpha}$ lines of $\mathrm{MbNO}$ and deoxyMb. There is an intensity decrease and slight blue shift and broadening of the $\mathrm{K}_{\alpha 1}$ line with increasing spin, as reported for other compounds ${ }^{24}$, while the $\mathrm{K}_{\alpha 2}$ line undergoes an intensity decrease and a slight broadening. Because $K_{\alpha}$ XES spectra of reference compounds are lacking, we resorted to DFT to simulate them (see Supplementary Note 4), as discussed later. In summary, the above XES spectra for the Mb LS hexacoordinated and the HS pentacoordinated forms provide the two extreme cases before and after photodissociation of the ligand, expected in time-resolved experiments, which allow us to identify the intermediate steps.

The laser-off and laser-on $K_{\beta}$ spectra are shown in Fig. 3 for time delays up to $\sim 1.4 \mathrm{ps}$, while the inset zooms into the region of the maximum of the $K_{\beta 1,3}$ line. These spectra show an intensity decrease and blue shift of the latter, an intensity decrease in the $7050-7055 \mathrm{eV}$ region and a slight increase of the $\mathrm{K}_{\beta}$, line in the $7045-7050 \mathrm{eV}$ region, all of which reflect the trends predicted for a change from LS to HS Mb (Supplementary Fig. 3a) and qualitatively agree with reference Fe(II) complexes (Supplementary Fig. 3b and c) ${ }^{22}$. Laser-on spectra at later times are shown in Supplementary Fig. 5, and they exhibit a return to the laser-off spectra on tens to hundreds of ps. Further insight into these changes is obtained from the normalized transient $\mathrm{K}_{\beta}$ XES spectra (excited minus ground state signal) shown in Supplementary Fig. 6a for time delays up to $1.36 \mathrm{ps}$, and Supplementary Fig. $6 \mathrm{~b}$ for time delays up to $100 \mathrm{ps}$. These 


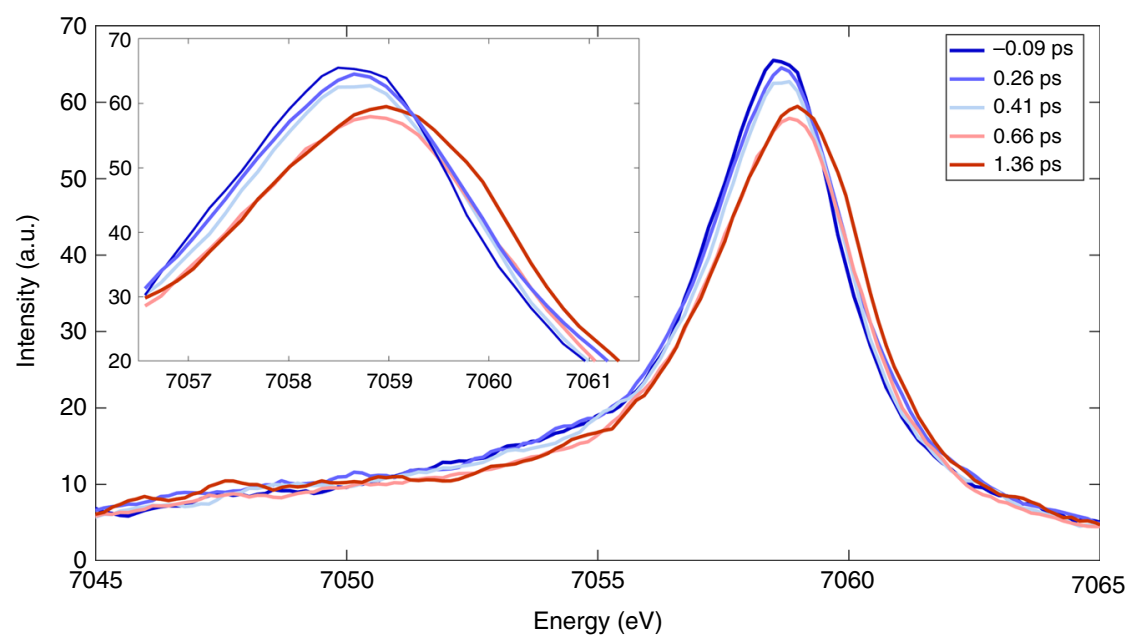

Fig. $3 \mathrm{~K}_{\boldsymbol{\beta}}$ XES spectra of MbNO at various time delays after visible photoexcitation. Laser-off ( $\left.-0.09 \mathrm{ps}\right)$ and Laser-on $\mathrm{K}_{\boldsymbol{\beta}} \mathrm{XES}$ spectra of MbNO at different time delays between 0.026 and $1.36 \mathrm{ps}$ (from blue to red) showing a blue shift of the $K_{\beta 1,3}$ line and an intensity decrease. The inset zooms into the region of the maximum of $K_{\beta_{1,3}}$ XES line at different time delays between -0.09 and $1.36 \mathrm{ps}$ (from blue to red) showing peak shifts smaller than the energy resolution $(\sim 0.5 \mathrm{eV})$ of our experiment.

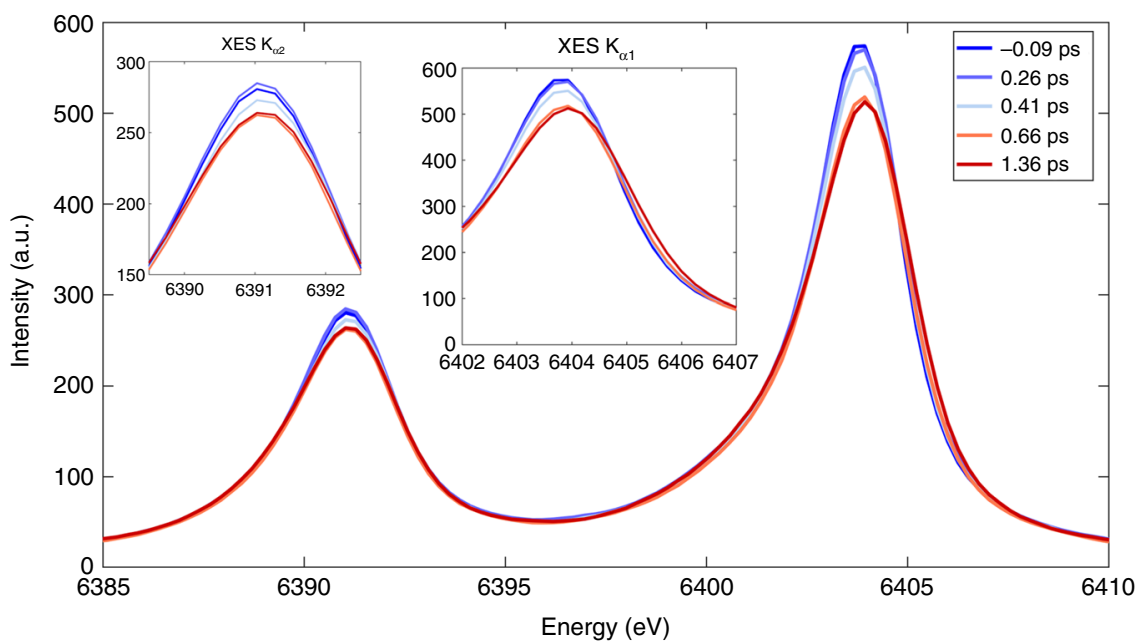

Fig. $4 \mathbf{K}_{\mathbf{\alpha}}$ XES spectra of MbNO at various time delays after visible photoexcitation. Laser-on ( -0.09 ps) and laser-om $K_{\alpha}$ XES spectra of MbNO at different time delays between 0.26 and $1.36 \mathrm{ps}$ (from blue to red) showing an intensity decrease for $\mathrm{K}_{\alpha 2}$ over the first $1.2 \mathrm{ps}$ after excitation and an intensity decrease and change in peak width for $\mathrm{K}_{\alpha \mathrm{\alpha}}$. Also, the visibility of changes in peak width $(\sigma)$ smaller than the energy resolution ( $\left.0.6 \mathrm{eV}\right)$ can be well observed. The insets zoom into the peaks of the $K_{\alpha 2}$ and $K_{\alpha 1}$ laser-on (pumped) XES spectra of MbNO at different time delays between -0.09 and 1.36 ps (from blue to red) showing an intensity decrease for $\mathrm{K}_{\alpha 2}$ over the first $1.2 \mathrm{ps}$ after excitation and an intensity decrease and change in peak width for $\mathrm{K}_{\alpha 1}$. The changes in peak width $(\sigma)$ smaller than the energy resolution $(\sim 0.6 \mathrm{eV})$ can be well observed.

transients exhibit a derivative-like shape near $7058 \mathrm{keV}$, a negative signal that extends down to $7050 \mathrm{eV}$ and a positive one below in the region of the $\mathrm{K}_{\beta}$, line. Beyond $\sim 1 \mathrm{ps}$, the normalized transient spectra no longer change but before, the $7050-7057 \mathrm{eV}$ region shows a gradual increase of negative amplitude, while the peak near $7060-7062 \mathrm{eV}$ is most blue shifted at $0.26 \mathrm{ps}$, and then shifts slightly to the red and stabilizes at $7060 \mathrm{eV}$ for times $>1 \mathrm{ps}$. The evolution of the 7050-7057 eV region qualitatively reproduces the static difference spectra of reference Fe-complexes of different spins (Supplementary Figs. 7 and 19), that revealed the transient population of a triplet state in the photoinduced SCO of [Fe $\left.(\text { bpy })_{3}\right]^{2+22}$. From Fig. 3 and Supplementary Fig. 6a, we conclude that reaching the final $\mathrm{HS}$ deoxyMb state requires $\sim 1 \mathrm{ps}$ and proceeds via an intermediate state.

Figure 4 shows the laser-off and laser-on $\mathrm{K}_{\alpha 1}$ and $\mathrm{K}_{\alpha 2}$ XES lines at different time delays, while the insets zoom into the region of their maxima. One notices a clear gradual weakening and a slight broadening (and for $\mathrm{K}_{\alpha 1}$, a slight blue shift) of the bands within the first ps. The trends in energy, width and intensity of these lines are consistent with the differences we observe between $\mathrm{MbNO}$ and deoxyMb (Supplementary Fig. 4), but in Fig. 4, the evolution is gradual and on a similar time scale as for the $\mathrm{K}_{\beta}$ lines. Supplementary Fig. 8 shows the laser-on spectra at later times, and just as in Supplementary Fig. 6, they exhibit a return to the laser-off spectra on tens to hundreds of ps. In order to highlight the changes, Supplementary Fig. 9 shows the experimental $K_{\alpha}$ XES transients at 0.26 and 1.36 ps time delay, while those at intermediate and at later times are shown in Supplementary Fig. 10. The normalized transient line shapes reflect the broadening, shift and intensity changes of the asymmetric emission lines, which do not change beyond $\sim 1 \mathrm{ps,} \mathrm{by} \mathrm{which}$ time the system is in the HS state, as confirmed by the difference 
of the experimental steady-state spectra deoxyMb minus $\mathrm{MbNO}$ (Supplementary Fig. 9b). In summary, both the $\mathrm{K}_{\alpha}$ and $\mathrm{K}_{\beta}$ XES transients point to an intermediate state being populated prior to the HS deoxyMb state that is fully formed by $\sim 1$ ps.

X-ray emission kinetics. The signal around $7053 \mathrm{eV}$ in Fig. 3 and Supplementary Fig. 6 best differentiates the intermediate state from the initial excited LUMO state to the quintet state. As already mentioned, this is where the signature of an intermediate spin state was most pronounced in the previous study on $[\mathrm{Fe}$ $\left.(\text { bpy })_{3}\right]^{2+22}$. By plotting its amplitude as a function of time for the transients available up to $3.6 \mathrm{ps}$, we can get an estimate of the lifetime of the intermediate state, using a three-level kinetic model (Supplementary Note 10 and Supplementary Fig. 11), assuming $100 \%$ Q-state population at $t=0$ that decays to the triplet state in $\sim 100 \mathrm{fs}$, as concluded from ultrafast fluorescence up-conversion studies ${ }^{47}$. The fit yields a lifetime of $500 \pm 250 \mathrm{fs}$ for the intermediate state (Supplementary Figs. 12 and 13).

Further details into the ultrafast kinetics comes from the peak position of the laser-on $K_{\beta_{1.3}}$ line (Fig. 3 and Supplementary Fig. 5) and the full width at half maximum (FWHM) of the laseron $K_{\alpha 1}$ line (Fig. 4 and Supplementary Fig. 8), both of which are sensitive to spin. During the first ps, these spectra reflect a mixture of doublet, short-lived intermediate state and of the final quintet state, before the entire excited population settles in the latter. The $\mathrm{K}_{\beta 1,3}$ peak energy shift and the FWHM of the $\mathrm{K}_{\alpha 1}$ line are plotted in Fig. 5 for times up to 750 ps, while the inset zooms into the first $10 \mathrm{ps}$. The red trace is a fit of the experimental data using a function consisting of a rising component and a biexponential decay. The former corresponds to a risetime of $800 \pm 150 \mathrm{fs}$ (see Supplementary Note 6), significantly longer than our cross-correlation $(\sim 150 \mathrm{fs})$, while the decay components have time constants (pre-exponential factors) of $\tau_{1}=30 \pm 9 \mathrm{ps}$ $\left(A_{1}=0.6\right)$ and $\tau_{2}=1.5 \pm 0.9 \mathrm{~ns}\left(A_{2}=0.4\right)$. Interestingly, while the UV-visible and IR TA results are characterized by a prompt risetime and decay times of 5-20, 110-290 ps, and a weak ns component ${ }^{7,11,13,30-37}$, the present XES observables exhibit different kinetics with a rise of $\sim 800 \mathrm{fs}$ and decays of $\sim 30 \mathrm{ps}$ and ns's. As mentioned previously, the $\sim 30$ ps decay had been reported in the time-resolved resonance Raman studies by Kruglik et al. ${ }^{13}$, who attributed it to the relaxation from the domed HS deoxyMb-NO species to the LS planar MbNO. Given the spin sensitivity of XES our observation of the $\sim 30$ ps decay is fully consistent with this interpretation as it reflects the return of the system to the LS ground state. Since the trigger to this process is the binding of the NO ligand to deoxyMb, in principle the geminate recombination (in 5-20 and 110-250 ps) and the nongeminate recombination (ns component) should feed the $30 \mathrm{ps}$ component. In this scenario, the 5-20 ps component would appear as a rise time, while the $110-250 \mathrm{ps}$ and the ns components would show up as decay because they are ratedetermining to the $30 \mathrm{ps}$ component. However, due to the scatter of data points at early times in Fig. 5, we refrained from extracting such times from the fits, preferring the above phenomenological function, which captures the important features using a single rise time and a biexponential decay. In this respect, the relatively large pre-exponential factor of the ns decay component, compared to previous reports (see ref. ${ }^{37}$ and references therein), most likely reflects the fact that it includes both geminate (110-250 ps) and non-geminate (ns) recombination of NO ligands.

Coming back to the short time dynamics, we have yet to identify the intermediate state between the initial MbNO excited state and the HS deoxyMb. The changes occurring at early times (Fig. 3 and Supplementary Fig. 5) reflect the transient population of an intermediate, most likely an $S=1$ spin state, if we parallel the present results with those on photoexcited $\left[\mathrm{Fe}(\mathrm{bpy})_{3}\right]^{2+22}$ We further support the assignment of the intermediate state as having $S=1$ using DFT calculations, presented in Supplementary Note 4 and Supplementary Figs. 14, 15 and 20. However, in addition to its spin sensitivity, $K_{\beta}$ XES is also sensitive to metal-ligand covalency ${ }^{43-46}$, which represents the charge donation from the ligands to the metal and therefore, it has a significant influence on the nature of metal-ligand bonds. The degree of covalency is connected via the effective number of unpaired $3 d$ electrons to the magnitude of the exchange splitting. In $K_{\beta}$ XES, it manifests itself in, among others, the splitting between the $K_{\beta 1,3}$ and $K_{\beta}$, lines. Thus, covalency could also affect the transient spectra as a result of dissociating the NO ligand and of the relaxation to the HS state, which involves an elongation of

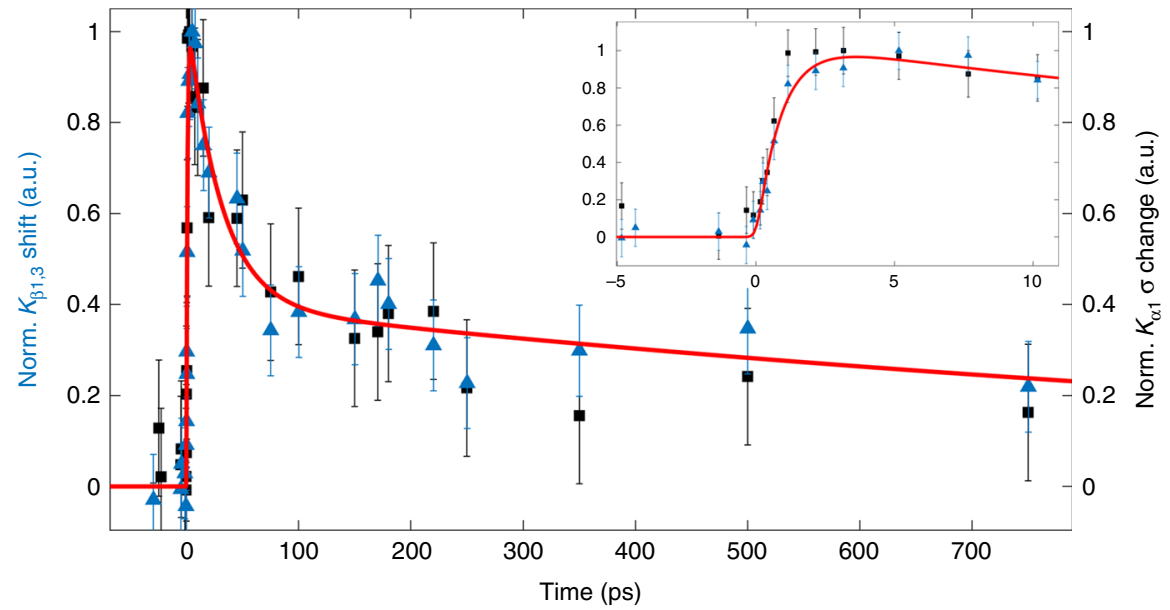

Fig. 5 Kinetic traces. Temporal evolution of the relative $K_{\beta_{13}}$ shift for MbNO (blue triangles, centroid obtained from Gaussian fit, max shift is $\sim 0.45$ eV, see Supplementary Note 6 ) and of the $K_{\alpha_{1}}$ peak width changes for MbNO (black squares, normalized $\sigma$ obtained from a Gaussian fit, maximum change in $\sigma$ is 0.3). The red line is a fit of the experimental data using a function consisting of a rising component and a biexponential decay. The former corresponds to a risetime of $800 \pm 150 \mathrm{fs}$ (see Supplementary Note 6 and Supplementary Fig. 18), significantly longer than our cross-correlation ( 150 fs), while the decay components have time constants (pre-exponential factors) of $\tau_{1}=30 \pm 9 \mathrm{ps}\left(A_{1}=0.6\right)$ and $\tau_{2}=1.5 \pm 0.9 \mathrm{~ns}\left(A_{2}=0.4\right)$. The inset shows the temporal evolution during the first 10 ps of both signals. The error bars represent the 1-sigma uncertainty of the Gaussian fit for the spectra at each time-point. Details are given in Supplementary Note 6 and Supplementary Fig. 18. 
the $\mathrm{Fe}-\mathrm{Np}$ bonds ( $\mathrm{Np}$ are the pyrrole nitrogens). We hold it however unlikely that covalency effects affect our results: (a) Given the recombination times of NO to deoxyMb (5-20 and $110-290 \mathrm{ps}$ ), it seems surprising that the $\mathrm{K}_{\beta}$ transient XES no longer changes beyond 1 ps (Supplementary Fig. 5). This is consistent with the $\mathrm{K}_{\beta}$ XES predominantly reporting on the spin state of the metal, i.e. since it does not distinguish between the HS deoxyMb and HS deoxyMb-NO species; (b) Also, the invariance of spectra in the relaxation from $\mathrm{NO}$-deoxyMb to planar $\mathrm{MbNO}$ in $\sim 30 \mathrm{ps}$ is to note, as this corresponds to a contraction of the $\mathrm{Fe}-\mathrm{Np}$ bonds; (c) Thus, neither NO rebinding nor the ensuing relaxation from domed to planar seem to affect the $\mathrm{K}_{\beta}$ XES. A fortiori, we would also exclude a contribution of covalency at early times $(<1 \mathrm{ps})$, since the reverse processes occur therein (ligand detachment, $\mathrm{Fe}-\mathrm{Np}$ bond elongation); (d) It should also be noted that the $K_{\beta}$ XES changes reported in the case of photoexcited $\left[\mathrm{Fe}(\mathrm{bpy})_{3}\right]^{2+}$, where a significant bond elongation occurs $^{48}$ towards the HS state, were fully interpreted in terms of transient population via an intermediate spin state based on reference spectra ${ }^{22}$; (f) Last but not least, covalency effects have not been reported on $\mathrm{K}_{\alpha}$ XES and the very close correspondence between the temporal evolutions of the $\mathrm{K}_{\alpha}$ and $\mathrm{K}_{\beta}$ XES (Fig. 5) suggests that signature of covalency is negligible in the latter.

To summarize our results, Fig. 6 shows the complete photocycle of MbNO: Upon photoexcitation into the Q-bands, prompt $(<100 \mathrm{fs})$ photodissociation of NO occurs leaving an excited pentacoordinated haem that undergoes doming by first relaxing to an intermediate spin state $(S=1)$, followed by a second relaxation step to the HS $S=2$ state forming deoxyMb. The entire cascade occurs in $\sim 800 \mathrm{fs}$. The relaxation back from the domed HS haem to the planar ground state occurs in $\sim 30 \mathrm{ps}$ upon NO recombination. The latter takes place over several time scales due to geminate (5-20, 110-200 ps) and non-geminate recombination (ns's). These results also show that the relaxation from domed $\mathrm{NO}-$ deoxyMb to the planar $\mathrm{MbNO}$ is a genuine $\mathrm{HS}$ to LS relaxation, as theoretically predicted by Franzen ${ }^{38}$, although constraints due to the F-helix (Fig. 1) ${ }^{13}$ cannot be fully excluded. Thus, the entire cycle of detachment and rebinding of NO to deoxyMb is accounted for by a SCO followed by a reverse SCO (Fig. 6). There are many parameters that affect the rates of forward-SCO and back-SCO but in the context of our interpretation, it is clear that the former is a stepwise process by jumps of $\Delta S=1$, which are ultrafast in metal complexes ${ }^{49}$, while the reverse $\mathrm{SCO}$ is a $\Delta S=2$ process occurring on a much slower time scale.

The above results have several implications on the description of the energy flow in haem proteins: (a) upon ligand detachment, the formation of the fully domed deoxyMb requires $\sim 800 \mathrm{fs}$, as it proceeds via an intermediate triplet state, which decays in $500 \pm$ $250 \mathrm{fs}$; (b) The ligand detachment is here mimicked by a photodissociation, which is prompt $(<50 \mathrm{fs})$, implying that the ensuing dynamics is taking place in the pentacoordinated haem and should therefore be the same for all ferrous haems involved in ligand release. This was already suggested by Ye et al. ${ }^{8}$ based on UV-visible TA studies, but they concluded that the ensuing evolution within the deoxyMb product is due to relaxation of a vibrationally hot species, while we conclude that it is an electronic relaxation cascade. The debate to determine the initial pathways for ultrafast energy flow in haem proteins, has been going one for over 30 years now, with the interpretation about formation of deoxyMb going from an electronic cascade via spin states ${ }^{5,6}$, to a vibrational energy redistribution ${ }^{8,16}$. Here by using elementspecific and spin-specific observables (XES), that are not sensitive to thermal and vibrational effects, we show that the initial scenario of a cascade among spin states is operative. Nevertheless, the cascade is non-radiative and energy gaps between spin states are dissipated in the form of heat to the environment. We believe that this behaviour is not limited to ferrous haems (that undergo

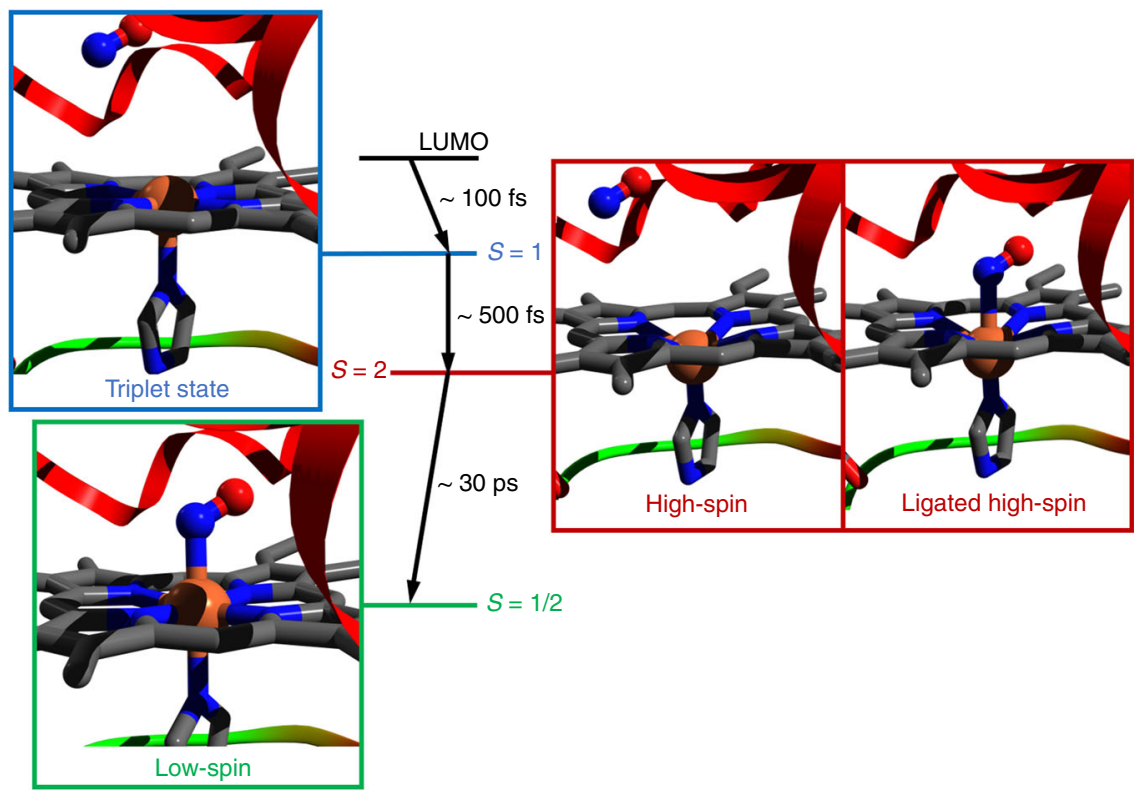

Fig. $\mathbf{6}$ Scheme of the spin cross-over (SCO) and reverse SCO characterizing the photocycle of MbNO. The low-spin ground state MbNO ( $S=1 / 2)$ haem (lower left panel) undergoes prompt dissociation of NO upon $\pi-\pi^{\star}$ excitation. The porphyrin Q-state decays to a triplet state ( $S=1$, upper left panel) of the pentacoordinated deoxyMb haem in $<100 \mathrm{fs}^{47}$. Further relaxation to the quintet state of the pentacoordinated haem occurs in $\sim 500 \pm 250 \mathrm{fs}$ (right "HighSpin" panel). The entire process (NO dissociation and relaxation from the Q-state to the high-spin quintet state) occurs in $~ 800 \mathrm{fs}$. Upon recombination of $\mathrm{NO}$ to deoxyMb leading to a hexacoordinated HS-domed haem (right "ligated high-spin" panel), relaxation back to the LS planar ground state occurs in $\sim 30 \mathrm{ps}$. The initial low spin structure is PDB entry 2FRJ ( $\mathrm{Fe}=$ orange, $\mathrm{N}=$ blue, $\mathrm{O}=$ red and $\mathrm{C}=$ grey), the sizes of the Fe atom, the $\mathrm{NO}$ molecule and the doming are exaggerated in order to highlight the key changes in the photocycle. The actual structural differences between MbNO and deoxyMb are shown in Supplementary Fig. 1. 
distal ligand dissociation) but it also includes ferric haems (that remain hexacoordinated) as suggested by the conclusions of UV-visible studies on metMb ${ }^{50}$ and $\mathrm{MbCN}^{51}$. Finally, doming has been suggested to trigger large-scale conformational changes of the protein, the so-called "protein quake", that opens a channel for ligand escape from the protein ${ }^{52}$. Our results show this is unlikely to be a very fast process, contrary to earlier claims that assumed it to occur on the time scale of the Fe-ligand bond breaking 53 .

The present work identifies the transition from the planar ligated haem involved in ligand release to the domed deoxy form as a SCO, which we believe is identical in all ferrous haem proteins. However, the ensuing events following ligand recombination are specific to the NO ligand and are characterized by a reverse SCO. This work also shows the power of ultrafast XES at unravelling exquisite details of the haem transformations upon ligand release and uptake in haem proteins.

\section{Methods}

Pump-probe XES measurements and analysis. Femtosecond hard XES measurements were carried out on $4 \mathrm{mM}$ solutions of $\mathrm{MbNO}$ in a physiological medium $(\mathrm{pH}=7)$ under inert conditions $\left(\mathrm{He}\right.$ or $\mathrm{N}_{2}$ atmosphere) at $\mathrm{BL} 3$ at SACLA ${ }^{40}$. The sample solution was delivered through a $0.2 \mathrm{~mm}$-thick round liquid jet and its integrity was continuously monitored by a mobile UV-vis spectrometer. A laser pulse at $533 \mathrm{~nm}$ with a $\sim 45 \mathrm{fs}$ FWHM was used to excite the Q-bands of $\mathrm{MbNO}$ in a near colinear geometry with the X-ray beam (pink, $8.168 \mathrm{keV}$ ). An energy-dispersive X-ray emission spectrometer (von Hamos geometry) and a $2 \mathrm{D}$ MPCCD detector are used to record the iron $2 p-1 s\left(\mathrm{~K}_{\alpha}\right)$ and $3 p-1 s\left(\mathrm{~K}_{\beta}\right)$ fluorescence spectra. A timing tool was used to measure the X-ray/optical relative arrival time fluctuations on a pulse to pulse basis and sort each shot by its relative arrival time ( $\sigma=150 \pm 40$, Supplementary Fig. 16). For the analysis of the $\mathrm{K}_{\beta}$ transients, we have used reference spectra of Fe-containing compounds from ref. ${ }^{22}$ (see Supplementary Figs. 3 and 17). For the $K_{\alpha}$ emission, reference spectra are lacking, and we relied on DFT simulated XES spectra for the ground state of MbNO and states of different spins for deoxyMb (see Supplementary Note 4 and Supplementary Fig. 4)

\section{Data availability}

Processed data used on the analysis and shown in the figures of this manuscript are available in the Supplementary Information. Raw $K_{\alpha}$ and $K_{\beta}$ XES data were generated at SACLA and the European XFEL large-scale facilities and are available in the repository: https://figshare.com/articles/dataset/Data_of_Figure_3_-_XES_Kbeta/12636470.

Received: 10 May 2020; Accepted: 21 July 2020;

Published online: 18 August 2020

\section{References}

1. Girvan, H. M. \& Munro, A. W. Heme sensor proteins. J. Biol. Chem. 288, 13194-13203 (2013).

2. Liebl, U., Lambry, J.-C. \& Vos, M. H. Primary processes in heme-based sensor proteins. Biochim. Biophys. Acta-Proteins Proteom. 1834, 1684-1692 (2013).

3. Perutz, M. F., Wilkinson, A. J., Paoli, M. \& Dodson, G. G. The stereochemical mechanism of the cooperative effects in hemoglobin revisited. Annu. Rev. Biophys. Biomol. Struct. 27, 1-34 (1998).

4. Haldane, J. \& Smith, J. L. The oxygen tension of arterial blood. J. Physiol. 20, 497-520 (1896).

5. Martin, J. et al. Femtosecond photolysis of CO-ligated protoheme and hemoproteins: appearance of deoxy species with a 350-fsec time constant. Proc. Natl Acad. Sci. USA 80, 173-177 (1983).

6. Petrich, J. W., Poyart, C. \& Martin, J. L. Photophysics and reactivity of hemeproteins - a femtosecond absorption study of hemoglobin, myoglobin, and protoheme. Biochemistry-Us 27, 4049-4060 (1988).

7. Ye, X., Demidov, A. \& Champion, P. M. Measurements of the photodissociation quantum yields of $\mathrm{MbNO}$ and $\mathrm{MbO}(2)$ and the vibrational relaxation of the six-coordinate heme species. J. Am. Chem. Soc. 124, 5914-5924 (2002).

8. Ye, X. O. et al. Investigations of heme protein absorption line shapes, vibrational relaxation, and resonance Raman scattering on ultrafast time scales. J. Phys. Chem. A 107, 8156-8165 (2003).

9. Vos, M. H. Ultrafast dynamics of ligands within heme proteins. $B B A$ Bioenergetics 1777, 15-31 (2008).
10. Lim, M., Jackson, T. A. \& Anfinrud, P. A. Binding of Co to myoglobin from a heme pocket docking site to form nearly linear Fe-C-O. Science 269, 962-966 (1995).

11. Kim, J., Park, J., Lee, T. \& Lim, M. Dynamics of geminate rebinding of NO with cytochrome $\mathrm{c}$ in aqueous solution using femtosecond vibrational spectroscopy. J. Phys. Chem. B 116, 13663-13671 (2012).

12. Petrich, J. W., Lambry, J. C., Poyart, C. \& Martin, J. L. Subpicosecond resonance Raman-spectroscopy of carbonmonoxy-hemoglobin-dynamics of heme-protein interactions and vibrational cooling. Biophys. J. 53, A281-a281 (1988).

13. Kruglik, S. G. et al. Picosecond primary structural transition of the heme is retarded after nitric oxide binding to heme proteins. Proc. Natl Acad. Sci. USA 107, 13678-13683 (2010).

14. Champion, P. M., Rosca, F., Ionascu, D., Cao, W. X. \& Ye, X. Rapid timescale processes and the role of electronic surface coupling in the photolysis of diatomic ligands from heme proteins. Faraday Discuss. 127, 123-135 (2004).

15. Negrerie, M., Cianetti, S., Vos, M. H., Martin, J.-L. \& Kruglik, S. G. Ultrafast heme dynamics in ferrous versus ferric cytochrome $\mathrm{c}$ studied by time-resolved resonance Raman and transient absorption spectroscopy. J. Phys. Chem. B 110, 12766-12781 (2006)

16. Ferrante, C., Pontecorvo, E., Cerullo, G., Vos, M. \& Scopigno, T. Direct observation of subpicosecond vibrational dynamics in photoexcited myoglobin. Nat. Chem. 8, 1137 (2016).

17. Franzen, S., Kiger, L., Poyart, C. \& Martin, J. L. Heme photolysis occurs by ultrafast excited state metal-to-ring charge transfer. Biophys. J. 80, 2372-2385 (2001).

18. Levantino, M. et al. Observing heme doming in myoglobin with femtosecond X-ray absorption spectroscopy. Struct. Dyn. 2, 041713 (2015).

19. Milne, C. J., Penfold, T. J. \& Chergui, M. Recent experimental and theoretical developments in time-resolved X-ray spectroscopies. Coord. Chem. Rev. 277, 44-68 (2014).

20. Falahati, K., Tamura, H., Burghardt, I. \& Huix-Rotllant, M. Ultrafast carbon monoxide photolysis and heme spin-crossover in myoglobin via nonadiabatic quantum dynamics. Nat. Commun. 9, 4502 (2018).

21. Glatzel, P. \& Bergmann, U. High resolution $1 s$ core hole X-ray spectroscopy in $3 d$ transition metal complexes-electronic and structural information. Coord. Chem. Rev. 249, 65-95 (2005).

22. Zhang, W. K. et al. Tracking excited-state charge and spin dynamics in iron coordination complexes. Nature 509, 345-+ (2014)

23. Mara, M. W. et al. Metalloprotein entatic control of ligand-metal bonds quantified by ultrafast x-ray spectroscopy. Science 356, 1276-1280 (2017).

24. Kawai, J., Suzuki, C., Adachi, H., Konishi, T. \& Gohshi, Y. Charge-transfer effect on the linewidth of Fe Ka X-ray fluorescence spectra. Phys. Rev. B 50, 11347-11354 (1994)

25. Brunori, M. Nitric oxide moves myoglobin centre stage. Trends Biochem. Sci. 26, 209-210 (2001).

26. Moncada, S. \& Higgs, A. The L-arginine-nitric oxide pathway. N. Engl. J. Med. 329, 2002-2012 (1993).

27. Bredt, D. \& Snyder, S. H. Nitric oxide: a physiologic messenger molecule. Annu. Rev. Biochem. 63, 175-195 (1994).

28. Pawloski, J. R., Hess, D. T. \& Stamler, J. S. Export by red blood cells of nitric oxide bioactivity. Nature 409, 622-626 (2001).

29. Fiorucci, S. et al. NCX-1015, a nitric-oxide derivative of prednisolone, enhances regulatory $\mathrm{T}$ cells in the lamina propria and protects against 2, 4, 6trinitrobenzene sulfonic acid-induced colitis in mice. Proc. Natl Acad. Sci. USA 99, 15770-15775 (2002)

30. Kholodenko, Y., Gooding, E. A., Dou, Y., Ikeda-Saito, M. \& Hochstrasser, R. M. Heme protein dynamics revealed by geminate nitric oxide recombination in mutants of iron and cobalt myoglobin. Biochemistry-Us 38, 5918-5924 (1999).

31. Shreve, A. P., Franzen, S., Simpson, M. C. \& Dyer, R. B. Dependence of NO recombination dynamics in horse myoglobin on solution glycerol content. $J$. Phys. Chem. B 103, 7969-7975 (1999).

32. Kim, S., Jin, G. \& Lim, M. Dynamics of geminate recombination of NO with myoglobin in aqueous solution probed by femtosecond mid-IR spectroscopy. J. Phys. Chem. B 108, 20366-20375 (2004).

33. Yoo, B.-K., Kruglik, S. G., Lamarre, I., Martin, J.-L. \& Negrerie, M. Absorption band III kinetics probe the picosecond heme iron motion triggered by nitric oxide binding to hemoglobin and myoglobin. J. Phys. Chem. B 116, 4106-4114 (2012).

34. Petrich, J. W. et al. Ligand-binding and protein relaxation in heme-proteinsa room-temperature analysis of $\mathrm{NO}$ geminate recombination. Biochemistry-Us 30, 3975-3987 (1991).

35. Zemojtel, T. et al. NO-bound myoglobin: Structural diversity and dynamics of the NO ligand. J. Am. Chem. Soc. 126, 1930-1931 (2004).

36. Ionascu, D. et al. Temperature-dependent studies of $\mathrm{NO}$ recombination to heme and heme proteins. J. Am. Chem. Soc. 127, 16921-16934 (2005). 
37. Silatani, M. et al. NO binding kinetics in myoglobin investigated by picosecond Fe K-edge absorption spectroscopy. Proc. Natl Acad. Sci. USA 112, 12922-12927 (2015)

38. Franzen, S. Spin-dependent mechanism for diatomic ligand binding to heme. Proc. Natl Acad. Sci. USA 99, 16754-16759 (2002).

39. Ishikawa, T. et al. A compact X-ray free-electron laser emitting in the subangstrom region. Nat. Photonics 6, 540-544 (2012).

40. Tono, K. et al. Beamline, experimental stations and photon beam diagnostics for the hard x-ray free electron laser of SACLA. New J. Phys. 15, 083035 (2013).

41. Morse, R. H. \& Chan, S. Electron paramagnetic resonance studies of nitrosyl ferrous heme complexes. Determination of an equilibrium between two conformations. J. Biol. Chem. 255, 7876-7882 (1980)

42. Schuth, N. et al. Effective intermediate-spin iron in O2-transporting heme proteins. Proc. Natl Acad. Sci. USA 114, 8556-8561 (2017).

43. Galakhov, V. R. et al. Degree of covalency of LiCoO2: X-ray emission and photoelectron study. Solid State Commun. 99, 221-224 (1996).

44. Wang, X., de Groot, F. M. \& Cramer, S. P. Spin-polarized x-ray emission of 3 $\mathrm{d}$ transition-metal ions: a comparison via $\mathrm{K} \alpha$ and $\mathrm{K} \beta$ detection. Phys. Rev. $B$ 56, 4553 (1997).

45. Lundberg, M. et al. Metal-ligand covalency of iron complexes from highresolution resonant inelastic X-ray scattering. J. Am. Chem. Soc. 135, 17121-17134 (2013).

46. Pollock, C. J., Delgado-Jaime, M. U., Atanasov, M., Neese, F. \& DeBeer, S. K $\beta$ mainline X-ray emission spectroscopy as an experimental probe of metal-ligand covalency. J. Am. Chem. Soc. 136, 9453-9463 (2014).

47. Bräm, O., Cannizzo, A. \& Chergui, M. Ultrafast broadband fluorescence upconversion study of the electronic relaxation of metalloporphyrins. J. Phys. Chem. A 123, 1461-1468 (2019).

48. Gawelda, W. et al. Structural determination of a short-lived excited iron(II) complex by picosecond x-ray absorption spectroscopy. Phys. Rev. Lett. 98, 057401-1-4 (2007).

49. Chergui, M. Ultrafast photophysics of transition metal complexes. Acc. Chem. Res. 48, 801-808 (2015).

50. Consani, C., Aubock, G., Bram, O., van Mourik, F. \& Chergui, M. A cascade through spin states in the ultrafast haem relaxation of met-myoglobin. J. Chem. Phys. 140, 025103-1-8 (2014).

51. Helbing, J. et al. Time-resolved visible and infrared study of the cyano complexes of myoglobin and of hemoglobin I from Lucina pectinata. Biophys. J. 87, 1881-1891 (2004).

52. Ansari, A. et al. Protein states and protein quakes. Proc. Natl Acad. Sci. USA 82, 5000-5004 (1985).

53. Franzen, S., Lambry, J. C., Bohn, B., Poyart, C. \& Martin, J. L. Direct evidence for the role of heme doming as the primary event in the cooperative transition of hemoglobin. Nat. Struct. Biol. 1, 230-233 (1994).

\section{Acknowledgements}

This work was supported by the Swiss NSF via the NCCR:MUST and grants 200020_169914 and 200021_175649 and the European Research Council Advanced Grant H2020 ERCEA 695197 DYNAMOX. C.B. and G.F.M. benefited from the
InterMUST Women Postdoc Fellowship. The backing of the technical staff at SACLA is highly acknowledged. The experiments were performed with the approval of the Japan Synchrotron Radiation Research Institute (JASRI; Proposal No. 2017B8048). T.P. acknowledge support from the Leverhulme Trust (RPG-2016-103). W.G. acknowledges financial support by European XFEL and partial financial support from the Polish National Science Centre (NCN) under SONATA BIS 6 Grant No. 2016/22/E/ST4/00543.

\section{Author contributions}

M.C. conceived the project. M.C., D.K., C.B., and O.C. designed the experiment. D.K., C.B., O.C., B.S., T.K.a., Y.O., H.I., T.I., J.N., C.H., S.K., M.Y. and N.K. performed the experiment at SACLA. D.K., C.B., O.C., B.S., G.F.M., J.M.B., F.A.L., W.G., P.Z., S.S., W.G., D.K.h., CBr recorded the steady-state XES at Eu-XFEL. D.K., C.B., J.R.R. and M.C analysed the data. T.P. and TKe performed the D.F.T. simulations. M.C., D.K., C.B., J.R.R., C.J.M., T.S., K.M., T.P., discussed the results and analysis and contributed to the interpretation. D.K. and M.C. wrote the manuscript. All authors read and contributed to the manuscript.

\section{Competing interests}

The authors declare no competing interests.

\section{Additional information}

Supplementary information is available for this paper at https://doi.org/10.1038/s41467020-17923-w.

Correspondence and requests for materials should be addressed to M.C.

Peer review information Nature Communications thanks the anonymous reviewers for their contribution to the peer review of this work.

Reprints and permission information is available at http://www.nature.com/reprints

Publisher's note Springer Nature remains neutral with regard to jurisdictional claims in published maps and institutional affiliations.

(c) (i) Open Access This article is licensed under a Creative Commons Attribution 4.0 International License, which permits use, sharing, adaptation, distribution and reproduction in any medium or format, as long as you give appropriate credit to the original author(s) and the source, provide a link to the Creative Commons license, and indicate if changes were made. The images or other third party material in this article are included in the article's Creative Commons license, unless indicated otherwise in a credit line to the material. If material is not included in the article's Creative Commons license and your intended use is not permitted by statutory regulation or exceeds the permitted use, you will need to obtain permission directly from the copyright holder. To view a copy of this license, visit http://creativecommons.org/ licenses/by/4.0/.

(C) The Author(s) 2020 